\title{
The Transverse versus the Sagittal Approach in First-Trimester Uterine Artery Doppler Measurement
}

This article was published in the following Dove Press journal: International Journal of Women's Health

\author{
Kiattisak Kongwattanakul (D) \\ Sukanya Chaiyarach' \\ Suppasiri Hayakangchat' \\ Kaewjai Thepsuthammarat $\mathbb{D}^{2}$ \\ 'Department of Obstetrics and \\ Gynecology, Faculty of Medicine, Khon \\ Kaen University, Khon Kaen, Thailand; \\ ${ }^{2}$ Clinical Epidemiology Unit, Faculty of \\ Medicine, Khon Kaen University, Khon \\ Kaen, Thailand
}

Objective: The uterine artery pulsatility index (UtA-PI) is an important marker for predicting and assessing the risk of various complications such as pre-eclampsia and fetal growth restriction. The measurement of UtA-PI in the first trimester is usually conducted via the sagittal approach. The aim of this study was to evaluate UtA Doppler measurement using the transverse approach in the first trimester.

Methods: This was a prospective observational study of 50 women with singleton pregnancy at between 11-13+6 weeks of gestation. Uterine artery (UtA) Doppler variables were measured using both the transverse and sagittal approach. The two approaches were compared in terms of time required to complete the measurements and early diastolic notch. The sample $t$-test and Wilcoxon rank sign test were used to analyze the outcomes when appropriate. Bland-Altman plots were used to determine the agreement between the two approaches. A $P$-value $<0.05$ was considered statistically significant. Intra-class correlation (ICC) was used to evaluate the reliability of measurements.

Results: There were a total of 50 pregnant women who participated in the study and completed the study protocol. The mean age of all subjects was 29.6 years, and 24 (48\%) were nulliparous. We observed no difference in terms of mean UtA-PI between the two approaches (sagittal: 2.04, transverse: 2.03; mean difference $0.01, \mathrm{CI}-0.01,0.04 ; \mathrm{p}>0.309$ ), nor in the means of any other UtA variables. However, there were differences between the two approaches in terms of early diastolic notch (sagittal: 11 , transverse: $13 ; p>0.999$ ) and the mean time required to complete the measurements (transverse: $21.7 \mathrm{~s}$, sagittal: $24.3 \mathrm{~s}$; $\mathrm{p}=0.001$ ). The intra-class correlation coefficients (ICCs) were $0.985,0.963$, and 0.988 for the right, left, and mean UtA-PIs respectively.

Conclusion: The transverse approach at a bladder depth of less than $5 \mathrm{~cm}$ performed better than the sagittal approach in the measurement of first-trimester uterine arteries. It may, thus, may be useful as a complementary approach in cases in which there is difficulty obtaining measurements using the sagittal approach.

Keywords: uterine artery, transverse, sagittal, Doppler, pulsatility index

\section{Introduction}

The uterine artery pulsatility index (UtA-PI) is an evaluation of the function and resistance of blood vessels to the uterus and placenta, ${ }^{1-3}$ and is an important marker for predicting and assessing the risk of various complications such as pre-eclampsia and fetal growth restriction. ${ }^{2-6}$ These conditions can affect both the mother and the fetus and lead to placental abruption, intraventricular hemorrhage, coagulopathy, or premature birth. ${ }^{7,8}$
Kongwattanakul

Department of Obstetrics and

Gynecology, Faculty of Medicine, Khon

Kaen University, Khon Kaen 40002

Thailand

Tel +66 43363030

Fax +6643348395

Email Kiattisak@kku.ac.th
International Journal of Women's Health 2019:1 I 629-635

629

DovePress $f$ in

http://doi.org/10.2147ulWH.S228619 
Use of the UtA-PI and early diastolic notch wave form $^{9-12}$ during the first trimester of pregnancy has a sensitivity of $47.8 \%$ and specificity of $92.1 \%$ for predicting pre-eclampsia before 34 weeks. ${ }^{5}$ In addition, if combined with maternal factors, mean arterial pressure, and serum placental growth factor, it has a detection rate of $100 \%$ (95\% CI, $80-100 \%$ ) for pre-eclampsia before 32 weeks, $75 \%$ (95\% CI, 62-85\%) for pre-eclampsia before 37 weeks, and $47 \%$ (95\% CI, 44-51\%) for pre-eclampsia at or after 37 weeks, with a $10 \%$ false-positive rate, ${ }^{13}$ making the UtA-PI measurement is highly useful in the clinical prediction of preeclampsia.

The Fetal Medicine Foundation (FMF) recommends using the sagittal approach as the standardized protocol for the measurement of UtA-PI at 11-13 weeks' gestation. ${ }^{14}$ However, we have found the visualization and measurement of UtA-PI using this method to be challenging, as it sometimes requires turning the transducer to the transverse view until the uterine arteries are identified at the level of the internal os. The transverse approach to UtA-PI measurement may be a viable alternative to the sagittal approach, as there is some evidence the two are equivalent in terms of reliability, reproducibility, and time required. ${ }^{15}$

The aim of this study was to evaluate the transverse approach to UtA doppler measurement and compare it with the conventional sagittal approach in the first-trimester screening.

\section{Materials and Methods}

\section{Participants}

We conducted a prospective observational study at the Khon Kaen University Faculty of Medicine Department of Obstetrics and Gynecology (Thailand). The study period was from May to November 2018. The inclusion criteria were singleton pregnancy, age 18-40 years, $11-13^{+6}$ weeks of gestation, and fetal crow-rump length between 45 and $84 \mathrm{~mm}$. Subjects were excluded if they exhibited any of the following conditions: congenital uterine structural anomalies, vaginal bleeding during the current pregnancy, or any fetal anomaly detected using ultrasonography.

The study protocol was approved by the Khon Kaen University Ethics Committee (HE611180). Eligible subjects signed an informed consent form prior to study participation. Baseline characteristics, obstetric histories and biophysical measurements were recorded for all of the women who participated.

\section{Measurement Protocols}

Consecutive bilateral measurements were taken of the ascending branch of the Uterine artery (UtA) via Doppler examination, which was performed using both the sagittal and transverse approach. The measurements were taken by a single obstetrician experienced in maternal-fetal medicine who had attended a preeclampsia screening course organized by the Fetal Medicine Foundation in London. The ultrasound machine used was a Voluson E8 Expert series (GE Healthcare, Milwaukee, WI, USA) with TA probe RAB 2-5D.

The sagittal approach was conducted using the standardized protocol recommended by The Fetal Medicine Foundation (FMF): ${ }^{15}$ a midsagittal view of the uterus and cervical canal was achieved and tilted laterally until the uterine arteries were visualized by color Doppler at the level of the internal os (Figure 1). A pulsed wave Doppler was used with the sampling gate set at $2 \mathrm{~mm}$ to cover the whole vessel, and the angle of insonation was less than $30^{\circ}$. The peak systolic velocity (PSV) of the UtA exceeded $60 \mathrm{~cm} / \mathrm{s}$ to ensure that the uterine artery. ${ }^{10}$

The transverse approach was conducted as follows: a midsagittal view of the uterus and cervical canal was identified in the same manner as with the sagittal approach. The transducer was turned $90^{\circ}$ and gently moved to obtain a transverse view of the level of both sides of the internal os by color Doppler (Figure 2). The criteria used for UtA measurement were the same as those used with the sagittal approach. Similar consecutive waveforms were measured twice, and the mean measurements from each approach were recorded.

\section{Statistical Analysis}

The two approaches were compared in terms of uterine artery Doppler variables (PI, pulsatility index; PSV, peak systolic velocity; RI, resistance index and EDV; end diastolic velocity), time required for each measurement, and early diastolic notch. The sample size was calculated using two dependent means (two-tailed test) with a mean uterine artery pulsatility index of 1.5 , standard deviation of 0.5 , and a sensitivity of $80 \% .{ }^{16}$ The sample $t$-test and Wilcoxon rank sign test were used to analyze the outcomes when appropriate. Bland-Altman plots were used to determine the agreement between the two approaches. A $P$-value $<0.05$ was considered statistically significant. The intraclass correlation (ICC) was used to evaluate the reliability of measurements. Statistical analysis was performed using 

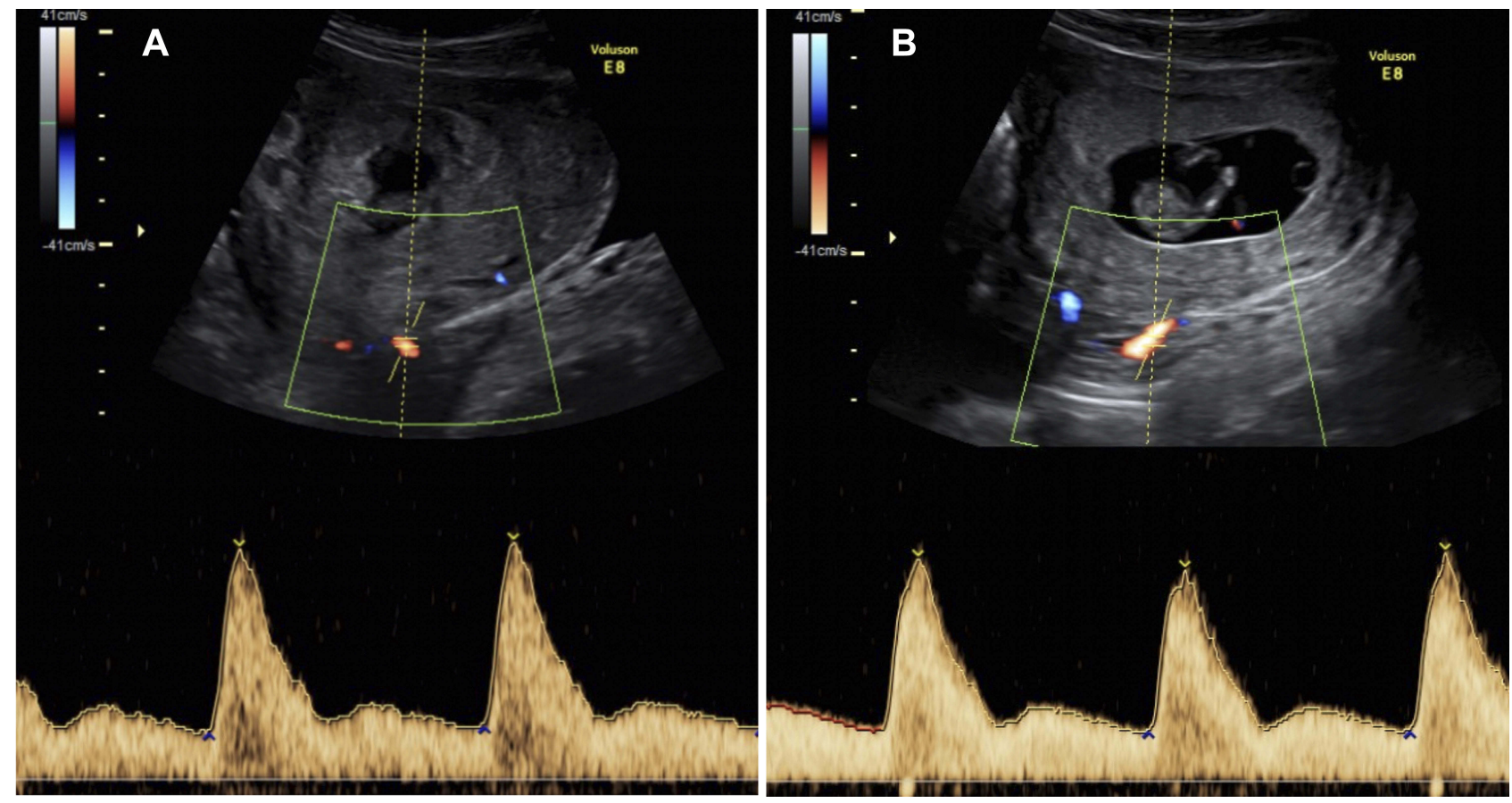

Figure I Uterine artery visualized by color Doppler at the level of the internal os using the sagittal approach with right (A) and left (B) waveforms.

Stata Statistical Software: Release 10 (StataCorp LP, College Station, TX).

\section{Results}

There were 56 eligible pregnant women recruited for this study, two of whom were excluded due to fetal anomalies (fetal megacystis, abdominal wall defect) and one due to multiple pregnancy. The uterine arteries could not be clearly visualized using the transverse approach in three of the participants.

In total, 50 women participated in the study and completed the study protocol. The mean age of all subjects was 29.6 years (range 18-40). The mean crown-rump length was $57.7 \mathrm{~mm}$ (SD 10.5, range 47.4-68.0 mm). Twentyfour $(48 \%)$ of the participants were nulliparous. Demographic data, including pre-pregnancy BMI, method of conception, smoking status, medical and obstetric history, biophysical measurements, and vertical bladder depth, are shown in Table 1.

Table 2 shows the UtA Doppler variables measured using the sagittal versus the transverse approach. We observed no difference in the mean UtA pulsatile index (UtA-PI; sagittal: 2.04, transverse: 2.03; mean difference 0.01 , CI $-0.01,0.04 ; \mathrm{p}>0.309$ ), nor in the means of any other UtA variables. However, there were differences between the two approaches in terms of early diastolic notch (sagittal: 11, transverse: $13 ; \mathrm{p}>0.999$ ) and the mean time required to complete the measurements (sagittal: $24.3 \mathrm{~s}$, transverse: $21.7 \mathrm{~s} ; \mathrm{p}=0.001$ ).

The Bland-Altman plots comparing right, left, and mean UtA-PI measurements between the two approaches are shown in Figure 3. Measurements taken using the sagittal approach were on average higher than those taken using the transverse approach. Overall bias of paired UtA-PI measurements obtained using the two approaches in the right and left UtAs and the mean of both arteries was 0.008 (95\% LoA, $-0.226,0.242)$, 0.020 (95\% LoA, $-0.375,0.414)$ and 0.014 (95\% LoA, $-0.177,0.204)$, respectively. The intra-class correlation coefficients (ICCs) were $0.985,0.963$, and 0.988 for the right, left, and mean UtA-PIs, respectively.

\section{Discussion}

The findings of this study indicate that the UtA-PI can be visualized adequately using the transverse approach with a strong concordance with results obtained using the conventional sagittal approach. In addition, the transverse approach was faster and better able to detect early diastolic notch when employed in first-trimester uterine artery Doppler measurement.

A previous study by Drouin et al on UtA Doppler measurement in 40 nulliparous participants found that the 

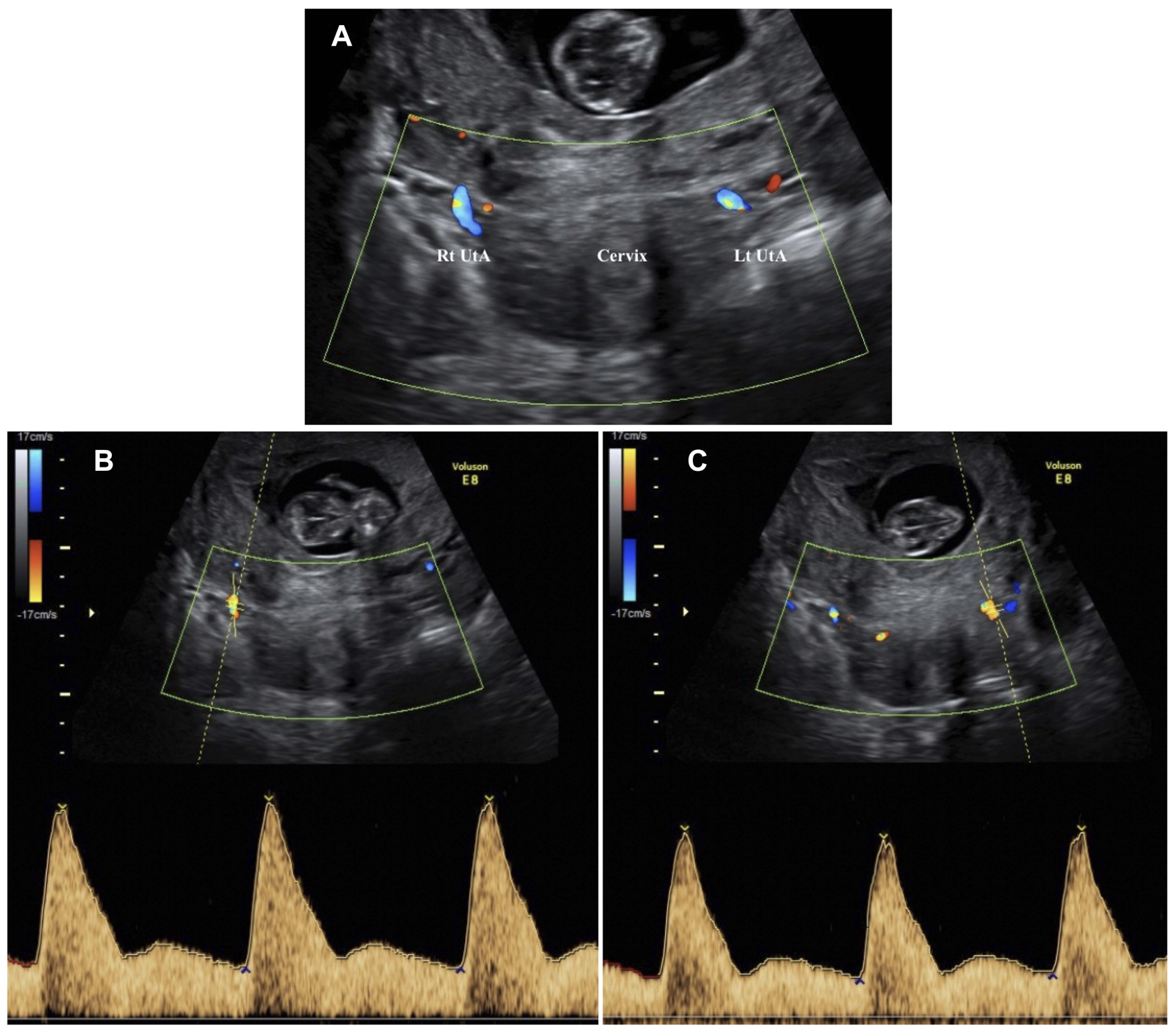

Figure 2 Uterine artery visualized by color Doppler at the level of the internal os using the transverse approach (A) with right (B) and left (C) Doppler measurement waveforms.

measurements taken using the sagittal approach were on average lower than those taken using the transverse approach. ${ }^{15}$ By contrast, our study found that the right, left and mean UtA-PIs obtained using the sagittal approach were slightly higher than those measured via the transverse approach. Nevertheless, the mean UtA-PI measurements in Drouin et al's study (sagittal: 2.11, transverse: 2.19 ) were similar to those found in our study (sagittal: 2.04, transverse: 2.03). In our hypothesis, it would be the effect of the pressure of the approach method. For the sagittal approach, there needs to apply pressure directly to the UtA on both sides. In contrast to the transverse approach, there pressed down to the internal os and less effect on the UtA.
There were three subjects in this study whose uterine arteries could not be visualized (failure rate: 5.7\%). In all three cases, the non-visualized arteries were on the left side, and the vertical bladder depth was greater than $5 \mathrm{~cm}$. One possible explanation for this is that the uterus is more inclined and rotated to the right due to displacement by the rectosigmoid colon on the left side during the first trimester, ${ }^{17}$ which may have made visualization at the level of the internal os using the transverse approach more difficult. Another possible explanation is that residual urine at a bladder depth of more than $5 \mathrm{~cm}$ might directly affect the uterine artery and indirectly from increase pressing the transducer. The results of this study confirmed that bladder 
Table I Characteristics of the Study Population

\begin{tabular}{|l|l|}
\hline Maternal Characteristics & $\mathbf{N}(\mathbf{5 0})$ \\
\hline Age (years) & $29.6(4.5)$ \\
Pre-pregnancy BMI $\left(\mathrm{kg} / \mathrm{m}^{2}\right)$ & $21 . \mathrm{I}(3.2)$ \\
Nulliparous, $\mathrm{n}(\%)$ & $24(48.0)$ \\
\hline Conception, $\mathrm{n}(\%)$ & \\
\hline Spontaneous & $47(94.0)$ \\
In-vitro fertilization & $3(6.0)$ \\
\hline Cigarette smoker, $\mathrm{n}(\%)$ & $2(4.1)$ \\
Family history of the patient had preeclampsia, $\mathrm{n}(\%)$ & $5(10.0)$ \\
Gestational age (weeks of gestation) & 12 (3/7 \\
Crown-rump length (mm) & $57.7(10.5)$ \\
\hline Medical history, $\mathrm{n}$ (\%) & \\
Chronic HT & $2(4.0)$ \\
DM & $2(4.0)$ \\
SLE & $3(6.0)$ \\
APS & $2(4.0)$ \\
Thyroid disease & $2(4.0)$ \\
\hline Obstetric history, $\mathrm{n}(\%)$ & \\
Previous early preeclampsia & $2(4.0)$ \\
Previous late preeclampsia & $1(2.0)$ \\
Previous spontaneous preterm birth & $5(10.0)$ \\
Previous fetal growth restriction & $3(6.0)$ \\
Previous stillbirth & 0 \\
\hline Biophysical measurements & $32.12(6.03)$ \\
\hline Systolic blood pressure (mmHg) & $116.56(9.85)$ \\
Diastolic blood pressure (mmHg) & $73.00(6.9 \mathrm{I})$ \\
Mean arterial pressure & $102.04(8.6 \mathrm{I})$ \\
\hline Bladder vertical depth (mm) & \\
\hline
\end{tabular}

Note: Data are given as $\mathrm{n}$, mean (SD) or $\mathrm{n}(\%)$.

depth should be less than $5 \mathrm{~cm}$ in order to visualize the structures at the level of the internal os using the transabdominal approach. ${ }^{18}$

The mean time required to complete the measurements for the transverse approach was faster than the sagittal approach because we can visualize and obtain UtA on both sides at the level of internal os at the same time. In contrast to the sagittal approach that needed to tilt laterally until the UtA is visualized by color Doppler at the level of the internal os.

Early diastolic notch represents reduced diastolic velocities and reflects abnormal uterine artery impedance. Notching has a low positive predictive value for preeclampsia and fetal growth restriction, in contrast to its $97 \%$ negative predictive value. ${ }^{19}$ In this study, the transverse approach detected diastolic notching slightly better
Table 2 Uterine Artery (UtA) Doppler Variables Measured Using the Sagittal and Transverse Approach in First Trimester of Pregnancy

\begin{tabular}{|c|c|c|c|}
\hline UtA Variable & $\begin{array}{l}\text { Sagittal } \\
\text { Approach }\end{array}$ & $\begin{array}{l}\text { Transverse } \\
\text { Approach }\end{array}$ & $\mathbf{P}$ \\
\hline \multicolumn{4}{|l|}{ UtA-PI } \\
\hline Right & $1.98(0.66)$ & $1.97(0.68)$ & 0.623 \\
\hline Left & $2.11(0.72)$ & $2.09(0.72)$ & 0.488 \\
\hline Mean & $2.04(0.61)$ & $2.03(0.64)$ & 0.309 \\
\hline \multicolumn{4}{|l|}{ UtA-RI } \\
\hline Right & $0.80(0.10)$ & $0.79(0.09)$ & 0.671 \\
\hline Left & $0.81(0.12)$ & $0.82(0.13)$ & 0.469 \\
\hline Mean & $0.80(0.10)$ & $0.81(0.10)$ & 0.665 \\
\hline \multicolumn{4}{|l|}{ UtA-PSV (cm/s) } \\
\hline Right & $88.35(20.65)$ & $90.94(20.49)$ & 0.133 \\
\hline Left & $86.45(19.08)$ & 88.69 (17.99) & 0.103 \\
\hline Mean & $87.40(15.98)$ & $89.66(15.12)$ & 0.093 \\
\hline \multicolumn{4}{|l|}{ UtA-EDV (cm/s) } \\
\hline Right & $19.62(9.33)$ & $20.25(10.03)$ & 0.241 \\
\hline Left & $18.59(10.85)$ & $20.11(10.63)$ & 0.148 \\
\hline Mean & $19.11(8.37)$ & $20.18(9.35)$ & 0.097 \\
\hline \multicolumn{4}{|c|}{ Early diastolic notch (n, \%) } \\
\hline Right & $5(10)$ & $6(12)$ & $>0.999$ \\
\hline Left & $6(12)$ & $7(14)$ & $>0.999$ \\
\hline \multicolumn{4}{|l|}{ Timing (seconds) } \\
\hline Right & $24.7(8.5)$ & $22.2(7.9)$ & 0.004 \\
\hline Left & $23.9(7.9)$ & $21.2(7.0)$ & 0.002 \\
\hline Mean & $24.3(7.8)$ & $21.7(7.2)$ & 0.001 \\
\hline
\end{tabular}

Note: Data are presented as mean (SD) or $\mathrm{n}(\%)$.

Abbreviations: $\mathrm{PI}$, pulsatility index; PSV, peak systolic velocity; RI, resistance index and EDV; end diastolic velocity.

than the sagittal approach, but this difference was not significant. Factors affecting early diastolic notch detection using the transverse approach, possible associations with adverse pregnancy outcomes, and the possible advantages of early screening should be evaluated in future studies.

The main strength of this study was that the measurements were performed by experienced obstetricians who are specialists in maternal-fetal medicine using a highquality ultrasound machine and FMF standardized protocol. Limitations of this study were that it was performed at a single center and did not investigate results obtained by less experienced sonographers. 

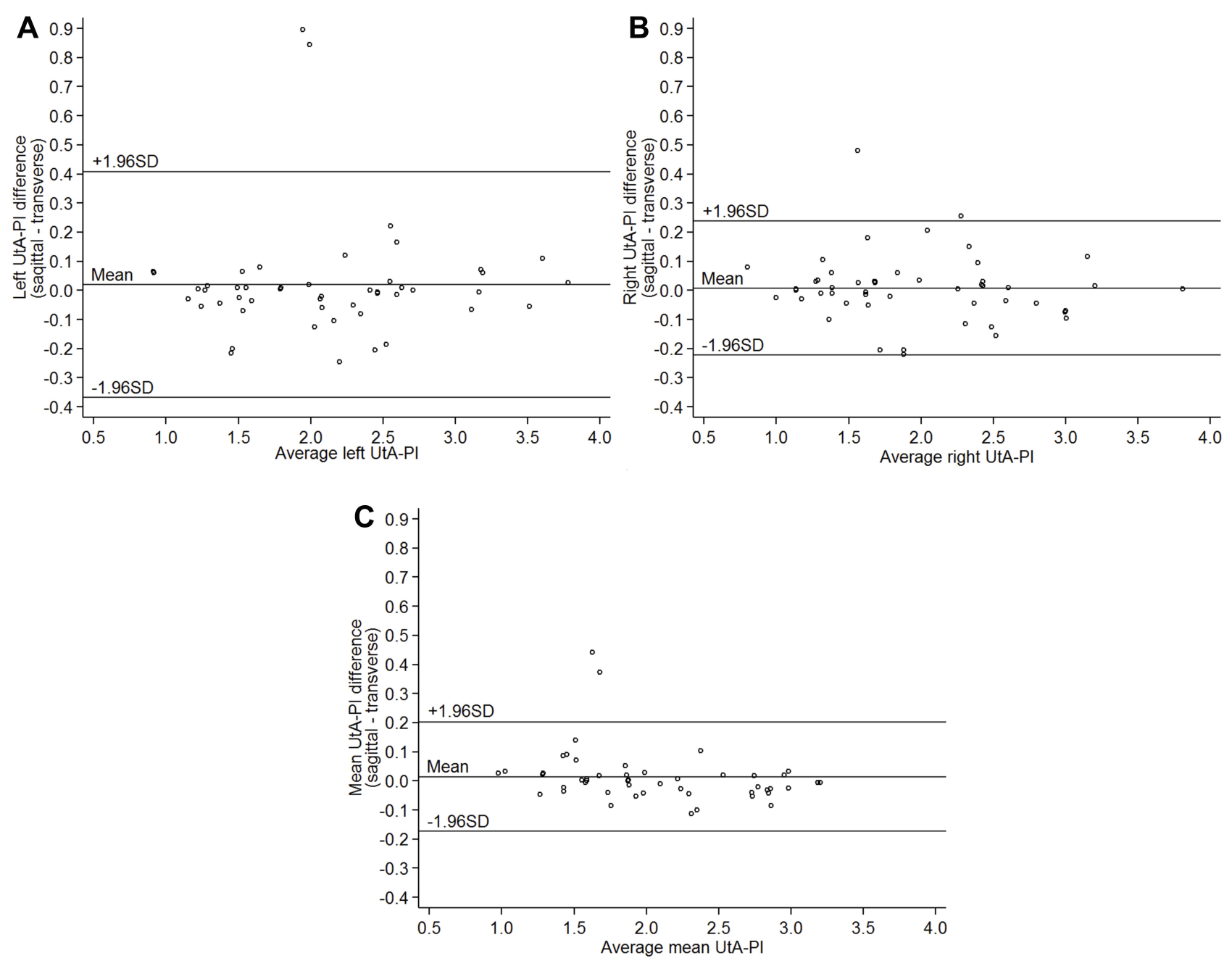

Figure 3 Bland-Altman plots for comparison of right (A), left, (B) and mean (C) uterine artery pulsatility indices (UtA-Pl) measured using the sagittal and transverse approach.

\section{Conclusion}

The transverse approach at a bladder depth of less than $5 \mathrm{~cm}$ performed well compared to the sagittal approach in the assessment of first-trimester uterine arteries and may be used as a complementary approach in cases in which there is difficulty obtaining measurements using the sagittal approach. Further studies are needed to evaluate the reproducibility and generalizability of these findings in cases in which the sonographer is less experienced, as well as factors correlated with failure to visualize the uterine artery.

\section{Details of Ethics Approval}

Ethical approvals were obtained from Khon Kaen University Ethics Committee for Human Research based on the Declaration of Helsinki and the ICH Good clinical Practice Guidelines. (17th May 2018, Reference No. HE611180).

\section{Acknowledgments}

We would like to thank Dylan Southard, the English Consultant Khon Kaen University's Faculty of Medicine for his help in editing the English-language presentation of this manuscript.

\section{Disclosure}

The authors have no conflicts of interest to report.

\section{References}

1. Gómez O, Figueras F, Fernández S, et al. Reference ranges for uterine artery mean pulsatility index at 11-41 weeks of gestation. Ultrasound Obstet Gynecol off J Int Soc Ultrasound Obstet Gynecol. 2008;32 (2):128-132. doi:10.1002/uog.5315

2. Gómez O, Martínez JM, Figueras F, et al. Uterine artery Doppler at 11-14 weeks of gestation to screen for hypertensive disorders and associated complications in an unselected population. Ultrasound Obstet Gynecol off J Int Soc Ultrasound Obstet Gynecol. 2005;26 (5):490-494. doi:10.1002/uog.1976 
3. Martin AM, Bindra R, Curcio P, Cicero S, Nicolaides KH. Screening for pre-eclampsia and fetal growth restriction by uterine artery Doppler at 11-14 weeks of gestation. Ultrasound Obstet Gynecol off J Int Soc Ultrasound Obstet Gynecol. 2001;18(6):583-586. doi:10.1046/j.0960-7692.2001.00594.x

4. O'Gorman N, Tampakoudis G, Wright A, Wright D, Nicolaides KH. Uterine artery pulsatility index at 12, 22, 32 and 36 weeks' gestation in screening for pre-eclampsia. Ultrasound Obstet Gynecol off J Int Soc Ultrasound Obstet Gynecol. 2016;47(5):565-572. doi:10.1002/ uog. 15819

5. Velauthar L, Plana MN, Kalidindi M, et al. First-trimester uterine artery Doppler and adverse pregnancy outcome: a meta-analysis involving 55,974 women. Ultrasound Obstet Gynecol off J Int Soc Ultrasound Obstet Gynecol. 2014;43(5):500-507. doi:10.1002/ uog. 13275

6. Poon LCY, Karagiannis G, Leal A, Romero XC, Nicolaides KH. Hypertensive disorders in pregnancy: screening by uterine artery Doppler imaging and blood pressure at 11-13 weeks. Ultrasound Obstet Gynecol off J Int Soc Ultrasound Obstet Gynecol. 2009;34 (5):497-502. doi:10.1002/uog.7439

7. Say L, Chou D, Gemmill A, et al. Global causes of maternal death: a WHO systematic analysis. Lancet Glob Health. 2014;2(6):e323e333. doi:10.1016/S2214-109X(14)70227-X

8. Backes CH, Markham K, Moorehead P, Cordero L, Nankervis CA, Giannone PJ. Maternal preeclampsia and neonatal outcomes. J Pregnancy. 2011;2011:214365. doi:10.1155/2011/214365

9. Napolitano R, Rajakulasingam R, Memmo A, Bhide A, Thilaganathan B. Uterine artery Doppler screening for pre-eclampsia: comparison of the lower, mean and higher first-trimester pulsatility indices. Ultrasound Obstet Gynecol off J Int Soc Ultrasound Obstet Gynecol. 2011;37(5):534-537. doi:10.1002/uog.8848

10. Plasencia W, Maiz N, Poon L, Yu C, Nicolaides KH. Uterine artery Doppler at $11+0$ to $13+6$ weeks and $21+0$ to $24+6$ weeks in the prediction of pre-eclampsia. Ultrasound Obstet Gynecol off J Int Soc Ultrasound Obstet Gynecol. 2008;32(2):138-146. doi:10.1002/ uog. 5402
11. Lefebvre J, Demers S, Bujold E, et al. Comparison of two different sites of measurement for transabdominal uterine artery Doppler velocimetry at 11-13 weeks. Ultrasound Obstet Gynecol off J Int Soc Ultrasound Obstet Gynecol. 2012;40(3):288-292. doi:10.1002/ uog. 11137

12. Cnossen JS, Morris RK, Ter Riet G, et al. Use of uterine artery Doppler ultrasonography to predict pre-eclampsia and intrauterine growth restriction: a systematic review and bivariable meta-analysis. CMAJ Can Med Assoc J J Assoc Medicale Can. 2008;178(6):701711. doi:10.1503/cmaj.070430

13. O'Gorman N, Wright D, Syngelaki A, et al. Competing risks model in screening for preeclampsia by maternal factors and biomarkers at 11-13 weeks gestation. Am J Obstet Gynecol. 2016;214(1):103.e1103.e12. doi:10.1016/j.ajog.2015.08.034

14. The Fetal Medicine Foundation. Assessment of risk for preeclampsia (PE) [Internet]; 2016. Available from: https://fetalmedicine.org/ calculator.

15. Drouin O, Johnson J-A, Chaemsaithong $\mathrm{P}$, et al. The transverse technique; a complementary approach to the measurement of firsttrimester uterine artery Doppler. Ultrasound Obstet Gynecol off J Int Soc Ultrasound Obstet Gynecol. 2018;52(5):639-647.

16. Alves JAG, Silva BYDC, de Sousa PCP, Maia SB, Costa FDS. Reference range of uterine artery Doppler parameters between the 11th and 14th pregnancy weeks in a population sample from Northeast Brazil. Rev Bras Ginecol E Obstet Rev Fed Bras Soc Ginecol E Obstet. 2013;35(8):357-362. doi:10.1590/S010072032013000800004

17. Chard T, Lilford R. Basic Sciences for Obstetrics and Gynaecology. 5 ed. London: Springer; 1998:198.

18. Kongwattanakul K, Saksiriwuttho P, Komwilaisak R, Lumbiganon P. Short cervix detection in pregnant women by transabdominal sonography with post-void technique. J Med Ultrason 2001. 2016;43 (4):519-522. doi:10.1007/s10396-016-073E

19. Khong SL, Kane SC, Brennecke SP, da Silva Costa F. First-trimester uterine artery Doppler analysis in the prediction of later pregnancy complications. Dis Markers. 2015;2015:679730.

\section{Publish your work in this journal}

The International Journal of Women's Health is an international, peerreviewed open-access journal publishing original research, reports, editorials, reviews and commentaries on all aspects of women's healthcare including gynecology, obstetrics, and breast cancer. The manuscript management system is completely online and includes a very quick and fair peer-review system, which is all easy to use. Visit http://www.dovepress.com/testimonials.php to read real quotes from published authors. 